1 | Apelt et al. Human HMGN1 and HMGN2 are not required for TCR

\title{
Human HMGN1 and HMGN2 are not required for transcription- coupled DNA repair
}

Katja Apelt ${ }^{1}$, Iris Zoutendijk ${ }^{1}$, Dennis Y. Gout ${ }^{1}$, Diana van den Heuvel ${ }^{1}$, and Martijn S. Luijsterburg ${ }^{1, *}$

1 Department of Human Genetics, Leiden University Medical Center, Einthovenweg 20, 2333 ZC, Leiden, The Netherlands

Running title: HMGN1 is not involved in TCR

* Corresponding author: Martijn Luijsterburg (m.luijsterburg@lumc.nl) 
2 | Apelt et al. Human HMGN1 and HMGN2 are not required for TCR

\section{Summary}

Transcription-coupled repair (TCR) removes DNA lesions from the transcribed strand of active genes. Stalling of RNA polymerase II (RNAPII) at DNA lesions initiates TCR through the recruitment of the CSB and CSA proteins. The full repertoire of proteins required for human TCR - particularly in a chromatin context - remains to be determined. Studies in mice have revealed that the nucleosome-binding protein HMGN1 is required to enhance the repair of UV-induced lesions in transcribed genes. However, whether HMGN1 is required for human TCR remains unaddressed. Here, we show that knockout or knockdown of HMGN1, either alone or in combination with HMGN2, does not render human cells sensitive to UV light or Illudin S-induced transcription-blocking DNA lesions. Moreover, transcription restart after UV irradiation was not impaired in HMGN-deficient cells. In contrast, TCR-deficient cells were highly sensitive to DNA damage and failed to restart transcription. Furthermore, GFP-tagged HMGN1 was not recruited to sites of UV-induced DNA damage under conditions were GFP-CSB readily accumulated. In line with this, HMGN1 did not associate with the TCR complex, nor did TCR proteins require HMGN1 to associate with DNA damagestalled RNAPII. Together, our findings suggest that HMGN1 and HMGN2 are not required for human TCR. 
3 | Apelt et al. Human HMGN1 and HMGN2 are not required for TCR

\section{Introduction}

Nucleotide excision repair (NER) is a versatile DNA repair system that removes a wide range of helix-distorting DNA lesions from our genome. Many of these DNA lesions, including ultra-violet (UV) light-induced photolesions, as well as lesions inflicted by compounds such as Illudin $S$ and cisplatin potently block transcription ${ }^{1-3}$. Transcription-coupled repair (TCR) is a specialized NER sub-pathway that preferentially removes DNA lesions from actively transcribed DNA strands 4 .

The stalling of elongating RNA polymerase II (RNAPIIo) at DNA lesions was proposed to initiate the TCR pathway by triggering the recruitment of the Cockayne syndrome proteins, CSB and CSA, which, in turn, likely recruit the downstream NER machinery to repair these lesions ${ }^{5}$. Even though the precise molecular mechanism of TCR is far from understood, emerging evidence suggests that modulating chromatin structure is an important prerequisite for mounting an efficient cellular response to transcription-blocking DNA lesions ${ }^{6-10}$.

Packaging of genomic DNA by histone and non-histone proteins into chromatin complicates efficient DNA repair ${ }^{11}$. Even though it could be argued that there is no need for modulating chromatin structure during TCR since chromatin is already rendered permissive to enable transcription ${ }^{12}$, several studies have implicated a role for chromatin-modifying activities associated with TCR-dependent transcription restart in human cells, including histone chaperones FACT and HIRA, and chromatin remodeling factor SNF2 $\mathrm{H}^{6-10}$. Furthermore, core TCR protein CSB is an ATPase with the ability to remodel nucleosomes in vitro ${ }^{13}$, which could contribute to TCR in vivo ${ }^{14}$.

Studies in mouse embryonic fibroblasts have revealed that the nucleosomebinding HMGN1 protein has the ability to increase the cellular transcription potential by unfolding higher-order chromatin structure ${ }^{15}$. Interestingly, mouse embryonic fibroblasts deficient in HMGN1 show a decreased repair rate of UV-induced DNA lesions particularly in transcribed genes ${ }^{16}$, suggesting an involvement in modulating chromatin structure during murine TCR. Rather than a specific TCR factor, murine HMGN1 seems to have a more general role in enabling DNA repair in chromatin, since mouse embryonic fibroblasts deficient in HMGN1 show not only defects in the repair of UV-induced lesions ${ }^{16}$, but also in the repair of oxidative DNA lesions ${ }^{17}$, and DNA double-strand breaks ${ }^{18}$. 
4 | Apelt et al. Human HMGN1 and HMGN2 are not required for TCR

Most studies addressing the versatile roles of HMGN1 have focused on mouse cells, while our current understanding of the function of HMGN1 in human cells is fairly limited. Although often assumed ${ }^{5}, 12$, experimental evidence showing that HMGN1 has a role in human TCR - similar to its murine counterpart - is lacking. In this study, we established human knockout cells for HMGN1 alone or in combination with HMGN2. Functional analysis revealed that, in contrast to mouse cells, human HMGN1 and HMGN2 are dispensable for human TCR. 
5 | Apelt et al. Human HMGN1 and HMGN2 are not required for TCR

\section{Results}

\section{Generation of human HMGN1 knockout cells}

Studies in mouse embryonic fibroblasts have revealed a role of HMGN1 in enhancing the repair rate of UV-induced DNA lesions in particular from transcribed genes ${ }^{16}$, suggesting a possible involvement of HMGN1 in murine transcription-coupled repair (TCR). However, whether HMGN1 is involved in human TCR has remained unexplored. To study a potential role of HMGN1 in human cells, we used CRISPR/Cas9-mediated genome editing to generate HMGN1 knock-out (KO) cells. To this end, we transfected U2OS cells with vectors encoding HMGN1-specific sgRNAs and Cas9 after which clones were isolated and screened (Figure 1A). Western blot analysis using HMGN1-specific antibodies confirmed the knock-out of HMGN1 in two independent clones (Figure 1A; clone 2-4 and 2-11). These findings reveal that loss of HMGN1 is viable in human cells and provide a new tool to study the role of HMGN1 in human cells.

\section{Human HMGN1-KO cells are resistant to Illudin S and UV}

Elongating RNA polymerase II (RNAPIIo) molecules are unable to efficiently bypass DNA lesions that block transcription, including those inflicted by the sesquiterpene drug Illudin $S^{2}$ or by ultra-violet (UV) light, which inflicts photoproducts such as cyclobutane pyrimidine dimers ${ }^{1}$. To overcome the obstacle posed by these DNA lesions, human cells fully depend on TCR to remove these lesions during transcription. Particularly Illudin S-induced lesions are remove exclusively by $\mathrm{TCR}^{2}$. To directly compare our HMGN1-KO cells in the same genetic background with TCR-deficient cells, we used CRISPR/Cas9-mediated genome editing to generate XPA knockout (KO) cells. Western blot analysis confirmed the knockout of XPA (Figure 1A). Moreover, clonogenic survivals assays in which cells were exposed to increasing concentrations of Illudin S (Figure 1B), or increasing doses of UV-C light (Figure 1C), confirmed that XPA-KO cells were highly sensitivity to these DNA-damaging agents compared to parental wild-type (WT) cells. In contrast, two independent HMGN1-KO clones, which, were included in parallel, showed no sensitivity to either Illudin S or UV irradiation compared to WT cells (Figure 1B, C). These findings show that, in contrast to murine cells ${ }^{16}$, loss of HMGN1 in human cells does not cause sensitivity to transcription-blocking DNA damage. 
6 | Apelt et al. Human HMGN1 and HMGN2 are not required for TCR

\section{Human HMGN1-KO cells show normal transcription recovery after UV}

In addition to sensitivity to transcription-blocking DNA damage, another hallmark of TCR-deficient cells is their inability to restart transcription after UV irradiation ${ }^{19}$. To quantify the ability of our HMGN1-KO cells to restart transcription, we performed recovery of RNA synthesis (RRS) experiments. To this end, we either mock treated or exposed cells to UV-C light $\left(6 \mathrm{~J} / \mathrm{m}^{2}\right)$. Nascent transcripts were pulse-labeled for 1 hour with the cell-permeable thymine analogue 5-ethynyl uridine (5-EU). Nascent transcripts containing 5-EU were visualized via Copper-catalyzed click chemistry of an azide-coupled fluorescent dye. Microscopic analysis revealed that WT cells showed a pronounced inhibition of transcription at 3 hours after UV, due to stalling of RNAPII, while significant transcription restart could be detected at 18 hours after UV irradiation (Figure 1D, E). This transcription restart was completely blocked in TCR-deficient XPA-KO cells due to their inability to clear transcription-blocking UV-induced lesions from the genome (Figure 1D, E). In contrast, two independent HMGN1-KO clones showed a normal restart of transcription after UV irradiation, suggesting that these cells are not deficient in TCR.

\section{HMGN2 does not compensate for HMGN1 in TCR}

Although HMGN1-deficient mouse embryonic fibroblasts are sensitive to UV irradiation ${ }^{16}$, we did not observe this phenotype in human HMGN1-KO cells. It has been reported that HMGN2 can functionally compensate for HMGN1 in murine cells ${ }^{20}$, and we therefore considered that a similar functional redundancy may mask the role of HMGN1 in human TCR. To test this possibility, we generated HMGN1/HMGN2 double knock-out (dKO) cells by CRISPR-Cas9-mediated genome editing. To this end, U2OS cells were co-transfected with vectors encoding sgRNAs targeting both HMGN1 and HMGN2 genes, as well as with a vector encoding the Cas9 protein. Cells were sorted by flow cytometry based on GFP expression encoded on the Cas9 vector, and clones were isolated and screened. Western blot analysis using antibodies specific for human HMGN1 and HMGN2 confirmed the loss of both HMGN proteins in our selected KO clones (Figure 2A; clones 1-5 and 1-6). Importantly, two independent HMGN1/HMGN2-dKO clones showed a normal transcription restart after UV irradiation in RRS experiments, while XPA-KO cells, included in parallel failed to resume transcription (Figure 2B, C). Furthermore, both HMGN1/HMGN2-dKO clones 
7 | Apelt et al. Human HMGN1 and HMGN2 are not required for TCR

were resistant to Illudin S-induced DNA lesions, while CSB-KO cells, which were included as a control, were highly sensitive to transcription-blocking lesions induced by this compound (Figure 2D). These findings suggest that HMGN2 does not functionally compensate for HMGN1, and that neither HMGN protein is required for TCR in human cells.

\section{Knockdown of HMGN1 or HMGN2 does not cause TCR defects in human cells}

Our previous findings using independently generated HMGN1-KO clones (Figure 1), or HMGN1/HMGN2-dKO clones revealed no signs of TCR deficiency (Figure 2). To rule out the possibility that these $\mathrm{KO}$ cells genetically adapted during their clonal expansion, we decided to employ a more acute way of removing the expression of HMGN proteins. To this end, we employed specific siRNAs to knockdown the expression of the HMGN proteins or XPA as a control. Western blot analysis showed that we achieved efficient knockdown of HMGN1, HMGN2, or XPA within a timecourse of four days (Figure 3A). However, as observed in our HMGN-KO cells, the knockdown of either HMGN1, HMGN2, or the combined knockdown of both HMGN proteins did not affect the restart of transcription after UV irradiation in RRS experiments, while knockdown of XPA fully impaired this process (Figure 3B, C). These findings suggest that acute knockdown of HMGN proteins, like genetic knockout of HMGN proteins, does not cause a deficiency in human TCR.

\section{Human HMGN1 does not associate with stalled RNAPII and TCR proteins}

Stalling of RNAPII at DNA lesions triggers the association of TCR proteins, including CSB, to initiate repair. We decided to monitor the possible association of HMGN1 with TCR proteins using two independent approaches. We first employed irradiation of cells with a pulsed $266 \mathrm{~nm}$ UV-C laser on a live-cell imaging set-up in which all glass optics were replaced by quartz optics to allow full UV-C transmission ${ }^{21}$. To monitor recruitment of proteins using this set-up, we stably expressed either GFP-CSB or HMGN1-GFP in the corresponding KO clones (Figure 4A). While UV-C laser-induced DNA damage readily triggered recruitment of GFP-CSB into locally irradiated regions, we failed to detect recruitment of HMGN1-GFP to sites of UV-C laser-induced DNA damage (Figure 4A). Importantly, the GFP-tagged human HMGN1 cDNA we used here, was previously shown to complement the phenotype of murine HMGN1-deficient cells demonstrating its functionality ${ }^{16}$. As an alternative approach, we employed 
8 | Apelt et al. Human HMGN1 and HMGN2 are not required for TCR

immunoprecipitation under native conditions after UV irradiation using the same cell lines that were used for live-cell imaging. While we could clearly detect a UV-induced association of RNAPIlo after immunoprecipitation of GFP-CSB, we failed to detect the association of endogenous HMGN1 under the same conditions (Figure 4B). Similarly, immunoprecipitation of GFP-tagged RNAPII from cells stably expressing GFP-RPB1 22, revealed robust UV-induced interactions with both CSB and CSA (Figure 4C), demonstrating that our conditions do allow us to detect interactions with TCR proteins after UV irradiation. However, we could not detect an interaction between GFP-RPB1 and endogenous HMGN1 under these conditions (Figure 4C). Reciprocal immunoprecipitation experiments on HMGN1-GFP also did not show any interactions with CSB, CSA, RNAPIIo, or HMGN2 in unirradiated or UV-irradiated cells (Figure 5A). Finally, we immunoprecipitated endogenous RNAPIlo, which strongly interacted with CSB, CSA and the TFIIH complex after UV irradiation (Figure 5B). However, while all these interactions with RNAPIlo were abolished in CSB-KO cells (Figure 5B), in line with the essential role of this protein in TCR, the UV-induced association of these TCR proteins with RNAPIlo was not affected in two independent HMGN1/HMGN2-dKO clones (Figure 5B). These findings show that human HMGN1 does not interact with DNA damage-stalled RNAPII and associated TCR proteins, and that both HMGN1 and HMGN2 are dispensable for human TCR. 
9 | Apelt et al. Human HMGN1 and HMGN2 are not required for TCR

\section{Discussion}

Although often inferred, based on studies in mouse embryonic fibroblasts ${ }^{16}$, it is currenly unknown if the nucleosome-binding protein HMGN1 has a role in modulating chromatin structure to enhance transcription-coupled DNA repair (TCR) in human cells. In the current study, we generated human HMGN1 knockout (KO) cells to directly adress this unanswered question. Functional analysis of human HMGN1-KO cells revealed that this nucleosome-binding protein is dispensible for human TCR. Our findings suggest that the role of murine HMGN1 in TCR is not functionally conserved in human cells.

\section{Human HMGN1 and HMGN2 are not involved in TCR}

Several key reviews on TCR mention HMGN1 and list this nucleosome-binding protein as a key factor that modulates human $\mathrm{TCR}^{5,12,23}$. However, it should be emphasized that while HMGN1-deficient mouse embryonic fibroblasts show decreased repair of UV-induced DNA lesions from active genes ${ }^{16}$, a functional role of HMGN1 in human TCR has not been experimentally adressed. Our results show that knockout or knockdown of the HMGN1 gene, either alone or in combination with the related HMGN2 gene, does not impair TCR in human cells. Several functional assays were performed to monitor a functional role in TCR, including clonogenic survival assays after exposure to either Illudin S or UV light, which both trigger transcription-blocking DNA lesions, or recovery of RNA synthesis (RRS) assays, which measure the ability of cells to restart transcription following UV irradiation. While cells knockout for either the XPA and CSB genes, which are essential for TCR, displayed pronounced defects in all these assays, all the HMGN1-KO or HMGN1/2-dKO clones were indistinguishable from wild-type cells. Thus, our results strongly suggest that HMGN1 and HMGN2 are not required for human TCR.

\section{Human HMGN1 does not associate with stalled RNAPIlo or TCR proteins}

Immunoprecipitation experiments do not support an association of HMGN1 with DNA damage-stalled RNAPIlo, or with either CSB or CSA in response to UV irradiation. These findings are in line with our funtional analysis, and strongly suggest that HMGN1 is not part of the TCR complex. Under the same conditions, we could readily detect a strong UV-induced association between RNAPIlo, CSB, CSA and the TFIIH complex, 
10 | Apelt et al. Human HMGN1 and HMGN2 are not required for TCR

arguing that our exerimental conditions would allow us to detect the association of HMGN1 if it would occur. Nonetheless, we could neither detect endogenous HMGN1 in CSB precipitates, nor could we detect TCR factors in HMGN1-GFP preciptates. Thus, our interaction experiments do not support an association of HMGN1 with the human TCR complex. Moreover, we show that the association of known TCR factors with DNA damage-stalled RNAPIlo is not affected by the combined loss of the HMGN1 and HMGN2 genes.

\section{Genetic differences between human and murine TCR}

Human HMGN1 is a small (100 amino acid) protein with striking sequence conservation (83\%) compared to mouse HMGN1 (96 amino acids; Supplemental Figure 1A). In fact, the sequence conservation between human and mouse HMGN1 (83\%) is far greater than that between human HMGN1 and HMGN2 (47\%; Supplemental Figure 1B, C). However, our findings suggest that human HMGN1 is not required for human TCR, while a previous study reported that UV-induced lesions in transcribed genes are repaired with decreased efficiency in HMGN1-deficient mouse cells ${ }^{16}$. Importantly, the UV-sensitive phenotype of these HMGN1-KO mouse cells could be rescued by re-expression of wild-type human HMGN1, but not by mutants that are either unable to bind to nucleosomes, or unable to unfold chromatin, suggesting that this phenotype is a specific effect of the loss of the HMGN1 gene in mice ${ }^{16}$.

The strong evolutionary similarity (83\%) between human and mouse HMGN1 suggest that it is unlikely that the function of HMGN1 between these species is not conserved due to changes at the protein level. However, there are fundamental differences between the organization of TCR in humans compared to mice that are much more likely to underlie these species-specific differences. In humans, the global genome repair (GGR) sub-pathway of NER recognizes and removes UV-induced cyclobutane pyrimidine dimers (CPD) through the DDB2 damage-recognition factor ${ }^{24-}$ 27. Indeed, inherited mutations in DDB2 cause a predisposition to develop skin cancer $^{28}$. In contrast, mice are largely deficient in the removal of CPDs by GGR owing to very low expression levels of $\mathrm{DDB} 2^{29}$, and instead rely on TCR to remove CPDs from their genome during transcription. Consequently, TCR-deficient CSB-/- and CSA/- mice develop skin cancer ${ }^{30}, 31$ due to their inability to repair CPDs, which is not observed in human Cockayne syndrome (CS) patients ${ }^{32}$. Conversely, CS mice do not 
11 | Apelt et al. Human HMGN1 and HMGN2 are not required for TCR

display strong neurological features ${ }^{30,31}$, which is a defining hallmark of CS in human patients ${ }^{32}$, further illustrating differences between mice and man when it comes to TCR deficiency. We propose that human cells may not have a need for HMGN1-mediated chromatin modulation to remove CPDs during TCR, because these lesions are targeted by DDB2-mediated GGR. Indeed, several studies have revealed that DDB2 mediates higher-order chromatin unfolding at sites of UV-induced DNA damage ${ }^{33,34}$ similar to what has been proposed for HMGN1 in mouse cells ${ }^{15}$.

In conclusion, our findings strongly suggest that the role of murine HMGN1 in transcription-coupled DNA repair is not conserved in human cells. 
12 | Apelt et al. Human HMGN1 and HMGN2 are not required for TCR

\section{Experimental Procedures}

Cell lines. All human cells (listed in table 1) were cultured at $37^{\circ} \mathrm{C}$ in an atmosphere of $5 \% \mathrm{CO} 2$ in DMEM (Thermo Fisher Scientific) supplemented with penicillin/streptomycin (Sigma) and 10\% Fetal bovine serum (FBS; Bodinco BV). Parental U2OS (WT) cells were a gift from Andreas Ladurner ${ }^{35}$. U2OS (FRT) cells containing the Flp-In ${ }^{\mathrm{TM}} / \mathrm{T}-\mathrm{REX}{ }^{\mathrm{TM}}$ system (Thermo Fisher Scientific), were a gift from Daniel Durocher ${ }^{36}$. All cell lines tested negative for mycoplasma contamination.

Generation of knockout cell lines. To generate single knockouts, U2OS (FRT) cells were cotransfected with pLV-U6g-PPB encoding a guide RNA from the LUMC/Sigma-Aldrich sgRNA library (see table 2 for plasmids, table 3 for sgRNA sequences) targeting the HMGN1, CSB or XPA gene together with an expression vector encoding Cas9-2A-GFP (pX458; Addgene \#48138) using lipofectamine 2000 (Invitrogen). Transfected cells were selected on puromycin $(1 \mu \mathrm{g} / \mathrm{mL})$ for 3 days, plated at low density after which individual clones were isolated. To generate HMGN1/HMGN2 double knockouts, U2OS (WT) cells were co-transfected with pLV-U6g-PPB sgHMGN1-2 and pX458 sgHMGN2 also encoding Cas9-2A-GFP. Transfected cells were FACS sorted on BFP/GFP, plated at low density after which individual clones were isolated. Isolated knockout clones were verified by Western blot analysis and/or sanger sequencing. The absence of Cas 9 integration / stable expression was confirmed by Western blot analysis.

Knockdown of HMGN1 or HMGN2. Cells were transfected twice with siRNAs at 0 and 36 hrs and were typically analyzed $60 \mathrm{hrs}$ after the first transfection. All siRNA transfections (see table 4 for siRNA sequences) were performed with 40 siRNA duplexes using Lipofectamine RNAiMAX (Invitrogen) in OptiMEM without FBS.

Generation of stable cell lines. Selected knockout clones of CSB and HMGN1 (see table 1) were subsequently used to stably express GFP-CSB, or HMGN1-GFP by co-transfection of pCDNA5/FRT/TO-Puro plasmid encoding these genes $(5 \mu \mathrm{g})$, together with pOG44 plasmid encoding the Flp recombinase $(0.5 \mu \mathrm{g})$. After selection on $1 \mu \mathrm{g} / \mathrm{mL}$ puromycin and $4 \mu \mathrm{g} / \mathrm{mL}$ blasticidin $\mathrm{S}$, single clones were isolated and expanded. Clones were selected based on their near-endogenous expression level of GFP-tagged proteins compared to parental U2OS Flp-In/T-REx cells. Expression of these GFPtagged proteins was induced by the addition of $2 \mu \mathrm{g} / \mathrm{ml}$ Doxycycline for $24 \mathrm{hrs}$.

Plasmid constructs. To insert sgRNA sequences targeting HMGN2 into pX458, two oligonucleotides (see tables 3 and 5) were annealed in annealing buffer ( $100 \mathrm{mM} \mathrm{NaCl}, 50 \mathrm{mM} \mathrm{HEPES}$; pH 7,4) by boiling for $5 \mathrm{~min}$ in water after which the mixture was allowed to cool down to room temperature. Annealed oligonucleotides were inserted into Bbsl-digested pX458 plasmid. The Neomycin resistance gene in pcDNA5/FRT/TO-Neo (Addgene \#41000) was replaced with a Puromycin resistance gene. Fragments spanning GFP-N1 (clontech) including the multiple cloning site were inserted into 
13 | Apelt et al. Human HMGN1 and HMGN2 are not required for TCR

pcDNA5/FRT/TO-puro. The HMGN1 cDNA was inserted as an Xhol / BsrGI fragment into pcDNA5/FRT/TO-Puro-GFP-N1. All sequences were verified by sequencing.

Clonogenic survival assays. Parental and knockout cell lines were trypsinized, seeded at low density and mock-treated or exposed to an increasing dose of UV light $\left(0.5,1,2 \mathrm{~J} / \mathrm{m}^{2}\right.$ of UV-C $\left.266 \mathrm{~nm}\right)$ or an increasing dose of Illudin S (Santa cruz; sc-391575) for 72 h (15, 30, 60, 100 pg/mL). On day 10, the cells were washed with $0.9 \% \mathrm{NaCl}$ and stained with methylene blue. Colonies of more than 20 cells were scored. Survival experiments were conducted in triplicate and repeated at least three times.

Immunoprecipitation for Co-IP. Cells were UV irradiated $\left(20 \mathrm{~J} / \mathrm{m}^{2}\right)$ or mock treated and harvested 1 $\mathrm{h}$ after UV. Chromatin-enriched fractions were prepared by incubating the cells for 20 min on ice in IP150 buffer (50 mM Tris pH 7.5, $150 \mathrm{mM} \mathrm{NaCl}, 0.5 \%$ NP-40, 2 mM MgCl 2 with protease inhibitor cocktail (Roche)), followed by centrifugation, and removal of the supernatant. For GFP-IPS, the chromatinenriched cell pellets were subsequently lysed in IP-150 buffer supplemented with 500 U/mL Benzonase Nuclease (Novagen) for $1 \mathrm{~h}$ at $4^{\circ} \mathrm{C}$. For endogenous RNA pol II IPs, the chromatin-enriched cell pellets were lysed in IP-150 buffer supplemented with $500 \mathrm{U} / \mathrm{mL}$ Benzonase Nuclease (Novagen) and $2 \mu \mathrm{g}$ RNAPII-S2 (ab5095, Abcam) for $1 \mathrm{~h}$ at $4{ }^{\circ} \mathrm{C}$, followed by adding concentrated $\mathrm{NaCl}$ to increase the $\mathrm{NaCl}$ concentration to $300 \mathrm{mM}$ and incubation of another 30 minutes at $4{ }^{\circ} \mathrm{C}$. Protein complexes were pulled down by $1.5 \mathrm{~h}$ incubation with Protein A agarose beads (Millipore; endogenous RNA pol II IPs) or GFP-Trap®_A beads (Chromotek; GFP IPS). For subsequent analysis by Western blotting, samples were prepared by boiling in Laemmli-SDS sample buffer.

Western blot. Cells were spun down, washed with PBS, and boiled for 10 minutes in Laemmli buffer (40 mM Tris pH 6.8, 3.35\% SDS, 16.5\% glycerol, 0.0005\% Bromophenol Blue and 0.05M DTT). Proteins were separated on 4-12\% Criterion XT Bis-Tris gels (Bio-Rad, \#3450124) in NuPAGE MOPS running buffer (NP0001-02 Thermo Fisher Scientific), and blotted onto PVDF membranes (IPFL00010, EMD Millipore). Membranes were blocked with blocking buffer (Rockland, MB-070-003) for $2 \mathrm{~h}$ at RT, and probed with the indicated antibodies (listed in table 6). An Odyssey CLx system (LI-COR Biosciences) was used for detection.

\section{RNA recovery assay}

30,000 cells were seeded on $12 \mathrm{~mm}$ glass coverslips in 24-wells plates in DMEM with $1 \%$ FBS. After 24 hours, cells were irradiated with UV-C at a dose of $6 \mathrm{~J} / \mathrm{m}^{2}$ and incubated in conditioned medium for different time periods (0,3 and 20 hours) to allow DNA repair and to restart RNA synthesis. Following incubation, nascent RNA was labelled by incubating the cells with $400 \mu \mathrm{M}$ 5-ethynyluridine (5-EU; Jena Bioscience; CLK-N002-10,), which was then visualized with a click-iT mix consisting of 50mM Tris buffer

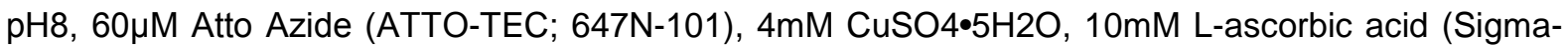
Aldrich; A0278) and 1:1000 DAPI (ThermoFisher; D1306) for one hour. Cell were washed three times for 5 minutes with PBS, and mounted on microscope slides (Thermo Scientific) using Aqua Polymount 
14 | Apelt et al. Human HMGN1 and HMGN2 are not required for TCR

(Polysciences, Inc. \#18606). All RRS experiments were conducted in triplicate and repeated at least three times.

Microscopic analysis of fixed cells. Images of fixed samples were acquired on a Zeiss Axiolmager M2 or D2 widefield fluorescence microscope equipped with 63x PLAN APO (1.4 NA) oil-immersion objectives (Zeiss) and an HXP 120 metal-halide lamp used for excitation. Fluorescent probes were detected using the following filters for DAPI (excitation filter: $350 / 50 \mathrm{~nm}$, dichroic mirror: $400 \mathrm{~nm}$, emission filter: 460/50 nm) and Alexa 555 (excitation filter: $545 / 25 \mathrm{~nm}$, dichroic mirror: $565 \mathrm{~nm}$, emission filter: 605/70 nm). Images were recorded using ZEN 2012 software and analyzed in Image J.

UV-C laser microscopy. Cells were grown on $18 \mathrm{~mm}$ Quartz coverslips and placed in a Chamlide CMB magnetic chamber in which growth medium was replaced by $\mathrm{CO}_{2}$-independent Leibovitz's $\mathrm{L} 15$ medium. Laser tracks were made by a diode pumped solid state $266 \mathrm{~nm}$ Yttrium Aluminum Garnet laser (Average power $5 \mathrm{~mW}$, repetition rate up to $10 \mathrm{kHz}$, pulse length $1 \mathrm{~ns}$ ) in a UGA-42-Caliburn/2L Spot Illumination system (Rapp OptoElectronic) with laser power set to $20 \%$. This was combined with live cell imaging in an environmental chamber set to $37^{\circ} \mathrm{C}$ on an all-quartz widefield fluorescence Zeiss Axio Observer 7 microscope, using a 100x 1.2 NA glycerol objective. The laser system is coupled to the microscope via a triggerbox and a neutral density (ND-1) filter is installed to block $90 \%$ of the laser light. A HXP $120 \mathrm{~V}$ metal-halide lamp was used for excitation. 
15 | Apelt et al. Human HMGN1 and HMGN2 are not required for TCR

\section{Table 1: Cell lines}

\begin{tabular}{|l|l|}
\hline Cell lines & Origin \\
\hline U2OS (FRT) & 36 \\
\hline U2OS (FRT) CSB-KO (1-12) & This study \\
\hline U2OS (FRT) CSB-KO (1-12)+ GFP-CSB (3) & This study \\
\hline U2OS (FRT) HMGN1-KO (1-9) & This study \\
\hline U2OS (FRT) HMGN1-KO (2-11) & This study \\
\hline U2OS (FRT) HMGN1-KO (2-4) & This study \\
\hline U2OS (FRT) HMGN1-KO (2-4) + HMGN1-GFP (9) & This study \\
\hline U2OS (FRT) XPA-KO (2-8) & This study \\
\hline U2OS (WT) & 35 \\
\hline U2OS (WT) EGFP-RPB1 & 22 \\
\hline U2OS (WT) HMGN1/HMGN2-dKO (1-5) & This study \\
\hline U2OS (WT) HMGN1/HMGN2-dKO (1-6) & This study \\
\hline
\end{tabular}

\section{Table 2: Plasmids}

\begin{tabular}{|l|l|}
\hline Plasmids & Origin \\
\hline pcDNA5/FRT/TO-Neo & Addgene \#41000 \\
\hline pcDNA5/FRT/TO-Puro & This study \\
\hline pcDNA5/FRT/TO-Puro-GFP-N1 & This study \\
\hline pcDNA5/FRT/TO-Puro-HMGN1-GFP & This study \\
\hline pEGFP-N1 & Clontech \\
\hline pHMGN1-EGFP & 16 \\
\hline pLV-U6g-PPB & LUMC/Sigma-Aldrich sgRNA library \\
\hline pLV-U6g-PPB sgCSB & This study \\
\hline pLV-U6g-PPB sgHMGN1-1 & This study \\
\hline pLV-U6g-PPB sgHMGN1-2 & This study \\
\hline pLV-U6g-PPB sgXPA & This study \\
\hline pOG44 & Thermo Fisher \\
\hline pX458 & Addgene \#48138 \\
\hline pX458 sgHMGN2-1 & This study \\
\hline pX458 sgHMGN2-2 & This study \\
\hline
\end{tabular}


16 | Apelt et al. Human HMGN1 and HMGN2 are not required for TCR

Table 3: Sequences of sgRNAs

\begin{tabular}{|l|l|}
\hline sgRNAs & Sequence \\
\hline sgCSB & CCAGACTTCAAGTCACAAAGTTG \\
\hline sgHMGN1-1 & GTAAGTCTTCTTTAGTTTCTTGG \\
\hline sgHMGN1-2 & CCTCAGTCTTCGTTTCCCCGTTT \\
\hline sgHMGN2-1 & TGCTAAGGGAGATAAAGCAA \\
\hline sgHMGN2-2 & GGGAGATAAAGCAAAGGTGA \\
\hline sgXPA & CCTGTGTCAATTATCTTTGGGGC \\
\hline
\end{tabular}

Table 4: Sequences of siRNAs

\begin{tabular}{|l|l|}
\hline siRNAs & Sequence \\
\hline siHMGN1-1 & GGATAAATCTTCAGACAAA \\
\hline siHMGN1-2 & TGATGAAGCAGGAGAGAAA \\
\hline siHMGN2-1 & AAGCAAAGGTGAAGGACGA \\
\hline siLuc & CGTACGCGGAATACTTCGA \\
\hline siXPA & CAGAGATGCTGATGATAAA \\
\hline
\end{tabular}

Table 5: Primers

\begin{tabular}{|l|l|}
\hline \multicolumn{2}{|l|}{ Primers } \\
\hline \multirow{2}{*}{$\begin{array}{l}\text { HMGN2-1 } \\
\text { (sgRNA) }\end{array}$} & CACCGTGCTAAGGGAGATAAAGCAA \\
\cline { 2 - 2 } $\begin{array}{l}\text { HMGN2-2 } \\
\text { (sgRNA) }\end{array}$ & AAACTTGCTTTATCTCCCTTAGCAC \\
\cline { 2 - 2 } & AAACTCACCTTTGCTTTATCTCCCC \\
\hline
\end{tabular}


17 | Apelt et al. Human HMGN1 and HMGN2 are not required for TCR

Table 6: Antibodies

\begin{tabular}{|l|l|l|l|l|l|}
\hline Antibodies & Host & & Clone & WB & \\
\hline Cas9 & Mouse & $\begin{array}{l}\text { Cell Signaling technology, } \\
\text { \#14697 }\end{array}$ & 7A9 and 3A3 & $1 / 5000$ & aML\#031 \\
\hline CSA/ERCC8 & Mouse & Santa Cruz, sc-376981 & D2 & $1 / 500$ & aML\#025 \\
\hline CSA/ERCC8 & Rabbit & Abcam, 137033 & EPR9237 & $1 / 750$ & aML\#028 \\
\hline CSB/ERCC6 & Goat & Santa Cruz, SC-10459 & E-18 & $1 / 1000$ & aML\#039 \\
\hline GFP & Mouse & Roche, \#11814460001 & 7.1 and 13.1 & $1 / 1000$ & aML\#011 \\
\hline GFP & Rabbit & Abcam, ab290 & & $1 / 1000$ & aML\#044 \\
\hline HMGN1 & Rabbit & Cell Signaling 5692 & \#5692 & $1: 1000$ & aML\#058 \\
\hline HMGN2 & Rabbit & Cell Signaling 9437 & & $1: 1000$ & aML\#065 \\
\hline $\begin{array}{l}\text { Mouse IgG (H+L) } \\
\text { CF770 }\end{array}$ & Goat & Biotium, VWR \#20077 & & $1 / 10000$ & aML\#009 \\
\hline p89/TFIIH & Mouse & Millipore, MABE1123 & 15TF2-1B3 & $1 / 2000$ & aML\#101 \\
\hline $\begin{array}{l}\text { rabbit IgG (H+L) } \\
\text { CF680 }\end{array}$ & Goat & Biotium, VWR \#20067 & & $1 / 10000$ & aML\#010 \\
\hline RNAPIIO & Rabbit & Abcam, ab5095 & & & \\
\hline Tubulin & Mouse & Sigma, T6199 & DM1A & $1 / 1000$ & aML\#008 \\
\hline XPA & Rabbit & kindly provided by Rick Wood & CJ1 & $1 / 10000$ & aML\#079 \\
\hline
\end{tabular}


18 | Apelt et al. Human HMGN1 and HMGN2 are not required for TCR

\section{Author Contributions}

KA generated knockout cells, performed clonogenic survivals, Western blot analysis to validate knockouts, Co-IP experiments, and wrote the paper. IZ generated single knockout cells and double knockout cells, performed clonogenic survivals, Western blot analysis to validate knockouts, and RRS experiments. DG generated double knockout cells, performed clonogenic survivals, Western blot analysis to validate knockouts, RRS experiments, and UV-C laser experiments. DVdH generated knockout cells, and Co-IP experiments. MSL performed UV-C laser experiments, supervised the project, and wrote the paper.

\section{Acknowledgments}

The authors acknowledge Rick Wood for his generous gift of antibodies. Andreas Ladurner provided U2OS (WT) cells, and Dan Durocher provided U2OS (FRT) cells. This work was funded by an LUMC Research Fellowship and an NWO-VIDI grant (ALW.016.161.320) to MSL.

\section{Competing interests}

The authors declare no conflict of interest.

\section{Data availability}

The datasets generated during and/or analysed during the current study are available from the corresponding author on reasonable request. 
bioRxiv preprint doi: https://doi.org/10.1101/835868; this version posted November 9, 2019. The copyright holder for this preprint (which was not certified by peer review) is the author/funder. All rights reserved. No reuse allowed without permission.

19 | Apelt et al. Human HMGN1 and HMGN2 are not required for TCR

\section{References}

1. Brueckner, F., Hennecke, U., Carell, T. \& Cramer, P. CPD damage recognition by transcribing RNA polymerase II. Science 315, 859-862 (2007).

2. Jaspers, N.G. et al. Anti-tumour compounds illudin S and Irofulven induce DNA lesions ignored by global repair and exclusively processed by transcription- and replication-coupled repair pathways. DNA Repair (Amst) 1, 1027-1038 (2002).

3. Damsma, G.E., Alt, A., Brueckner, F., Carell, T. \& Cramer, P. Mechanism of transcriptional stalling at cisplatin-damaged DNA. Nat Struct Mol Biol 14, 1127-1133 (2007).

4. Mellon, I., Spivak, G. \& Hanawalt, P.C. Selective removal of transcription-blocking DNA damage from the transcribed strand of the mammalian DHFR gene. Cell 51, $241-249$ (1987).

5. Marteijn, J.A., Lans, H., Vermeulen, W. \& Hoeijmakers, J.H. Understanding nucleotide excision repair and its roles in cancer and ageing. Nat Rev Mol Cell Biol 15, 465-481 (2014).

6. Dinant, C. et al. Enhanced chromatin dynamics by FACT promotes transcriptional restart after UV-induced DNA damage. Mol Cell 51, 469-479 (2013).

7. Wienholz, F. et al. FACT subunit Spt16 controls UVSSA recruitment to lesion-stalled RNA Pol II and stimulates TC-NER. Nucleic Acids Res 47, 4011-4025 (2019).

8. Aydin, O.Z. et al. Human ISWI complexes are targeted by SMARCA5 ATPase and SLIDE domains to help resolve lesion-stalled transcription. Nucleic Acids Res 42, 8473-8485 (2014).

9. Adam, S., Polo, S.E. \& Almouzni, G. Transcription recovery after DNA damage requires chromatin priming by the H3.3 histone chaperone HIRA. Cell 155, 94-106 (2013).

10. Oksenych, V. et al. Histone methyltransferase DOT1L drives recovery of gene expression after a genotoxic attack. PLoS Genet 9, e1003611 (2013).

11. Luijsterburg, M.S. \& van Attikum, H. Chromatin and the DNA damage response: the cancer connection. Mol Oncol 5, 349-367 (2011).

12. Hanawalt, P.C. \& Spivak, G. Transcription-coupled DNA repair: two decades of progress and surprises. Nat Rev Mol Cell Biol 9, 958-970 (2008).

13. Citterio, E. et al. ATP-dependent chromatin remodeling by the Cockayne syndrome B DNA repair-transcription-coupling factor. Mol Cell Biol 20, 7643-7653 (2000).

14. Cho, I., Tsai, P.F., Lake, R.J., Basheer, A. \& Fan, H.Y. ATP-dependent chromatin remodeling by Cockayne syndrome protein $B$ and NAP1-like histone chaperones is required for efficient transcription-coupled DNA repair. PLoS Genet 9, e1003407 (2013).

15. Bustin, M. Chromatin unfolding and activation by HMGN(*) chromosomal proteins. Trends Biochem Sci 26, 431-437 (2001).

16. Birger, Y. et al. Chromosomal protein HMGN1 enhances the rate of DNA repair in chromatin. EMBO J 22, 1665-1675 (2003).

17. Masaoka, A. et al. HMGN1 protein regulates poly(ADP-ribose) polymerase-1 (PARP-1) selfPARylation in mouse fibroblasts. J Biol Chem 287, 27648-27658 (2012).

18. Kim, Y.C. et al. Activation of ATM depends on chromatin interactions occurring before induction of DNA damage. Nat Cell Biol 11, 92-96 (2009).

19. Nakazawa, Y., Yamashita, S., Lehmann, A.R. \& Ogi, T. A semi-automated non-radioactive system for measuring recovery of RNA synthesis and unscheduled DNA synthesis using ethynyluracil derivatives. DNA Repair (Amst) 9, 506-516 (2010).

20. Deng, T. et al. Functional compensation among HMGN variants modulates the DNase I hypersensitive sites at enhancers. Genome research 25, 1295-1308 (2015).

21. Dinant, C. et al. Activation of multiple DNA repair pathways by sub-nuclear damage induction methods. J Cell Sci 120, 2731-2740 (2007).

22. Caron, P. et al. WWP2 ubiquitylates RNA polymerase II for DNA-PK-dependent transcription arrest and repair at DNA breaks. Genes \& development 33, 684-704 (2019).

23. Gerlitz, G. HMGNs, DNA repair and cancer. Biochim Biophys Acta 1799, 80-85 (2010).

24. Fitch, M.E. et al. The DDB2 nucleotide excision repair gene product $\mathrm{p} 48$ enhances global genomic repair in p53 deficient human fibroblasts. DNA Repair (Amst) 2, 819-826 (2003).

25. Fitch, M.E., Nakajima, S., Yasui, A. \& Ford, J.M. In vivo recruitment of XPC to UV-induced cyclobutane pyrimidine dimers by the DDB2 gene product. J Biol Chem 278, 46906-46910 (2003).

26. Scrima, A. et al. Structural basis of UV DNA-damage recognition by the DDB1-DDB2 complex. Cell 135, 1213-1223 (2008). 
bioRxiv preprint doi: https://doi.org/10.1101/835868; this version posted November 9, 2019. The copyright holder for this preprint (which was not certified by peer review) is the author/funder. All rights reserved. No reuse allowed without permission.

20 | Apelt et al. Human HMGN1 and HMGN2 are not required for TCR

27. Moser, J. et al. The UV-damaged DNA binding protein mediates efficient targeting of the nucleotide excision repair complex to UV-induced photo lesions. DNA Repair (Amst) 4, 571582 (2005).

28. Rapic-Otrin, V. et al. True XP group E patients have a defective UV-damaged DNA binding protein complex and mutations in DDB2 which reveal the functional domains of its p48 product. Human molecular genetics 12, 1507-1522 (2003).

29. Tan, T. \& Chu, G. p53 Binds and activates the xeroderma pigmentosum DDB2 gene in humans but not mice. Mol Cell Biol 22, 3247-3254 (2002).

30. van der Horst, G.T. et al. Defective transcription-coupled repair in Cockayne syndrome B mice is associated with skin cancer predisposition. Cell 89, 425-435 (1997).

31. van der Horst, G.T. et al. UVB radiation-induced cancer predisposition in Cockayne syndrome group A (Csa) mutant mice. DNA Repair (Amst) 1, 143-157 (2002).

32. Wilson, B.T. et al. The Cockayne Syndrome Natural History (CoSyNH) study: clinical findings in 102 individuals and recommendations for care. Genetics in medicine : official journal of the American College of Medical Genetics 18, 483-493 (2016).

33. Luijsterburg, M.S. et al. DDB2 promotes chromatin decondensation at UV-induced DNA damage. J Cell Biol 197, 267-281 (2012).

34. Adam, S. et al. Real-Time Tracking of Parental Histones Reveals Their Contribution to Chromatin Integrity Following DNA Damage. Mol Cell 64, 65-78 (2016).

35. Sellou, H. et al. The poly(ADP-ribose)-dependent chromatin remodeler Alc1 induces local chromatin relaxation upon DNA damage. Mol Biol Cell 27, 3791-3799 (2016).

36. Panier, S. et al. Tandem protein interaction modules organize the ubiquitin-dependent response to DNA double-strand breaks. Mol Cell 47, 383-395 (2012). 
A

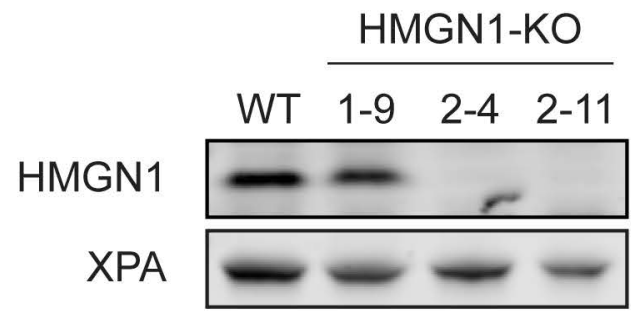

B

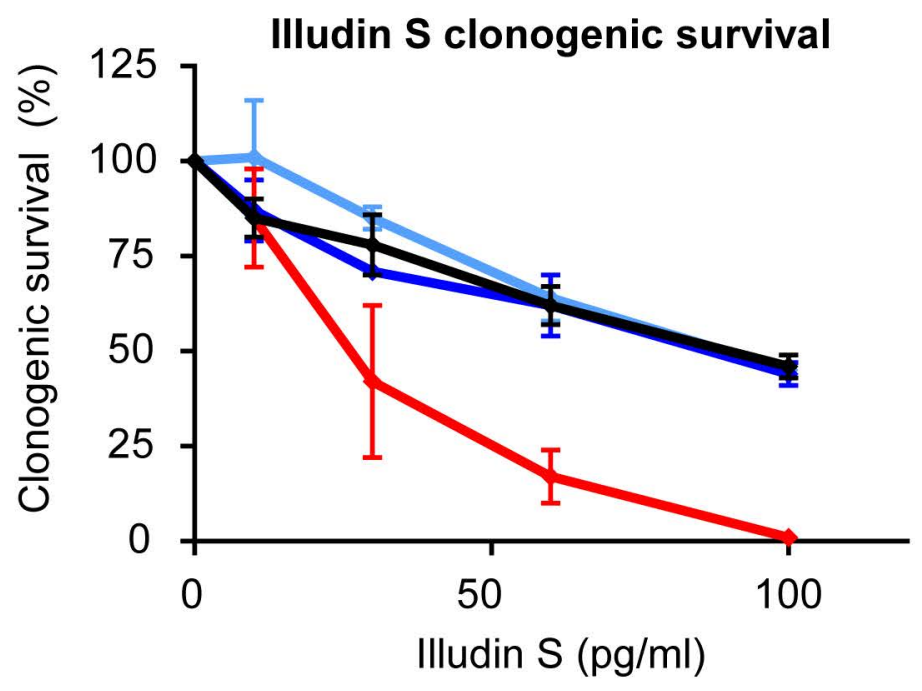

$$
\begin{array}{ll}
\rightarrow \text { WT } & \rightarrow \text { HMGN1-KO }(2-4) \\
\rightarrow \text { XPA-KO } & \rightarrow \text { HMGN1-KO }(2-11)
\end{array}
$$

D

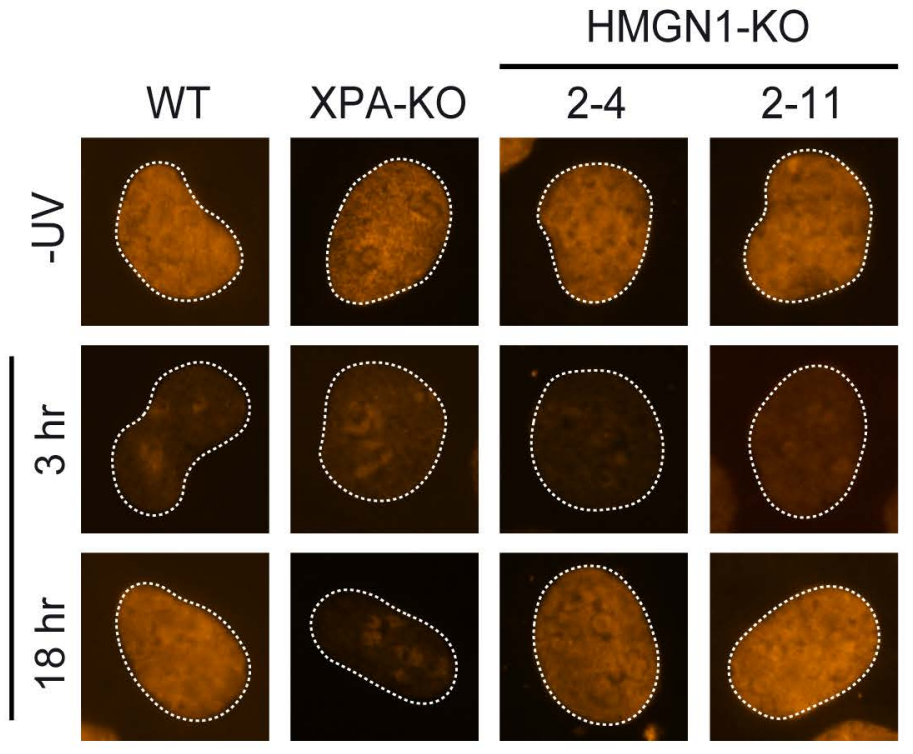

C

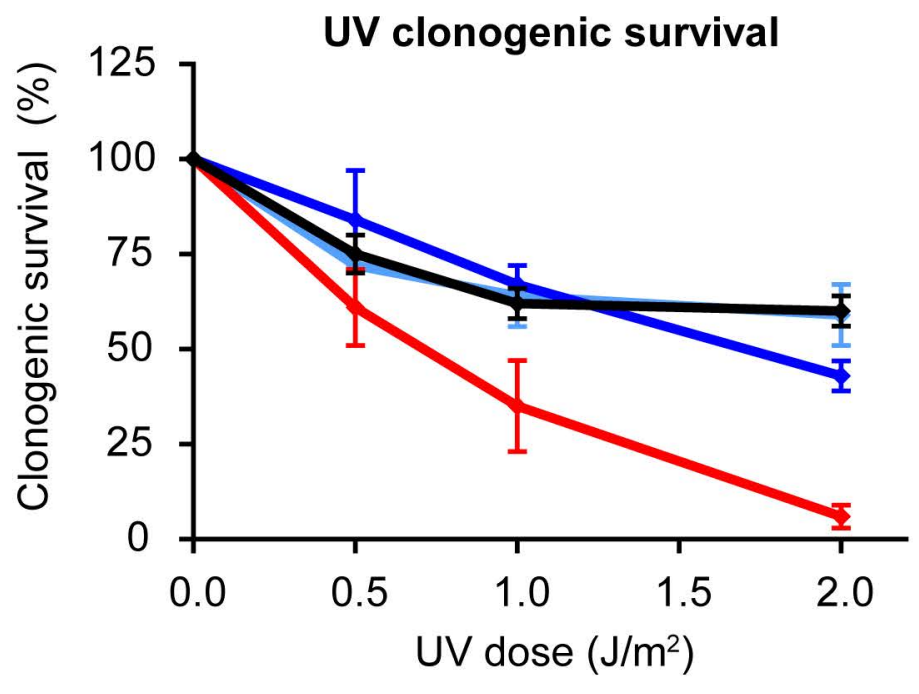

$\mathbf{E}$

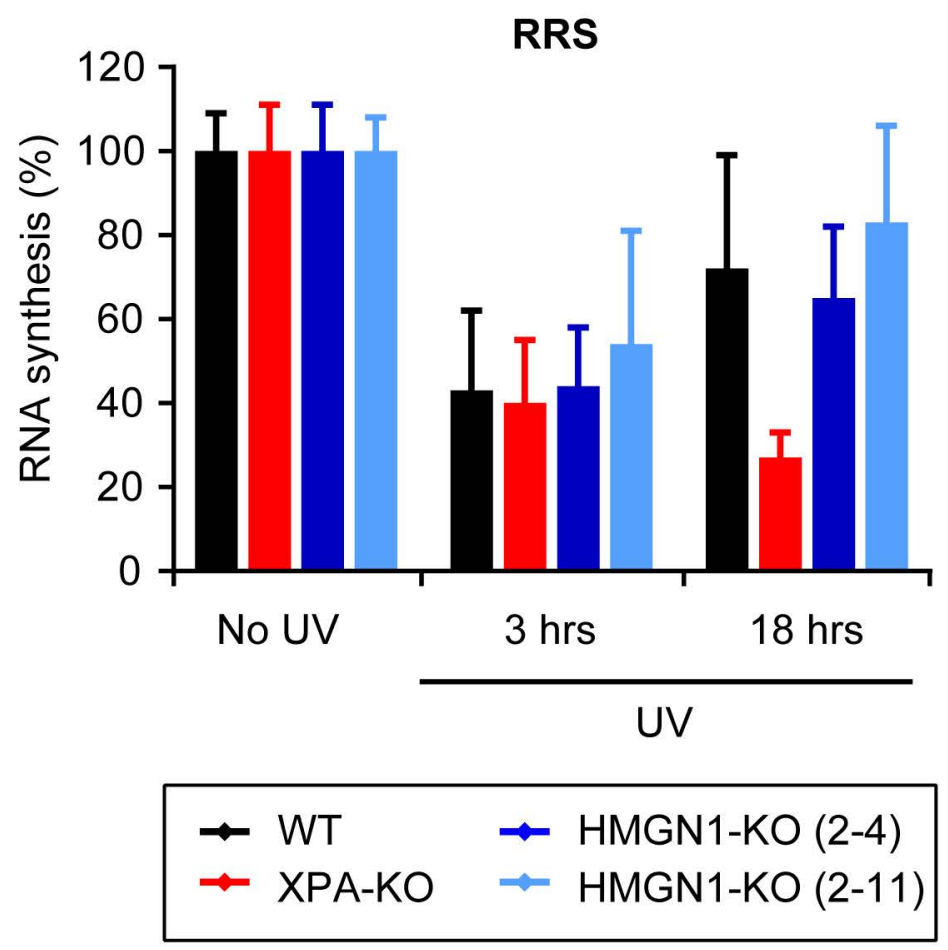


Figure 1. HMGN1 knockout does not impact human TCR. (a) Western blot analysis of U2OS WT and HMGN-KO clones or XPA-KO clone. (b) Clonogenic Illudin S survival or (c) clonogenic UV survival of WT, XPA-KO, and HMGN1-KO cell lines. Data represent mean \pm SEM of three independent experiments. (d) Representative microscopy images, and (e) Quantification of RRS after UV on the WT, XPA-KO, and HMGN1-KO cell lines. Data represent mean \pm SEM of three independent experiments. Uncropped Western blot data is shown in the Supplementary Information file. 
Figure bigRxiv preprint doi: https://doi.org/10.1101/835868; this version posted November 9, 2019. The copyright holder for this preprint (which

A was not certified by peer review) is the author/funder. All rights reserved. No reuse allowed without permission.

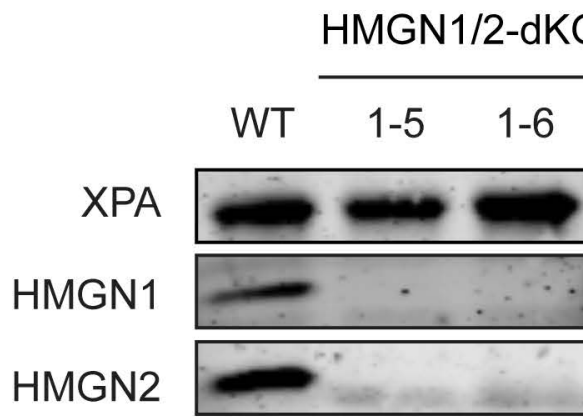

B

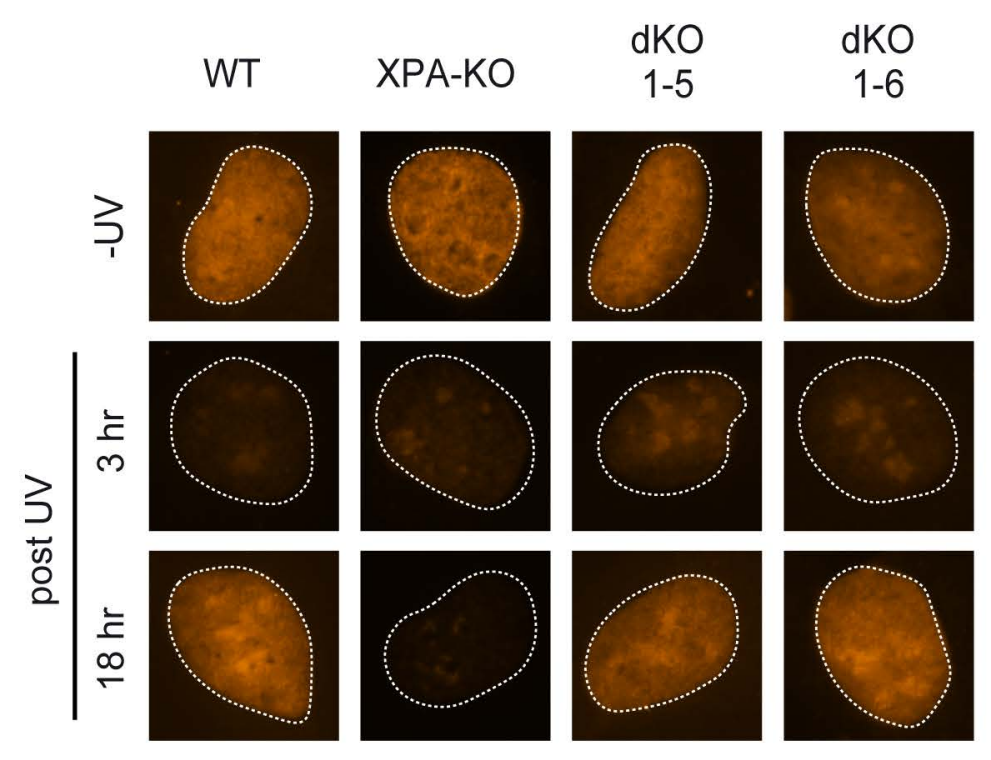

C
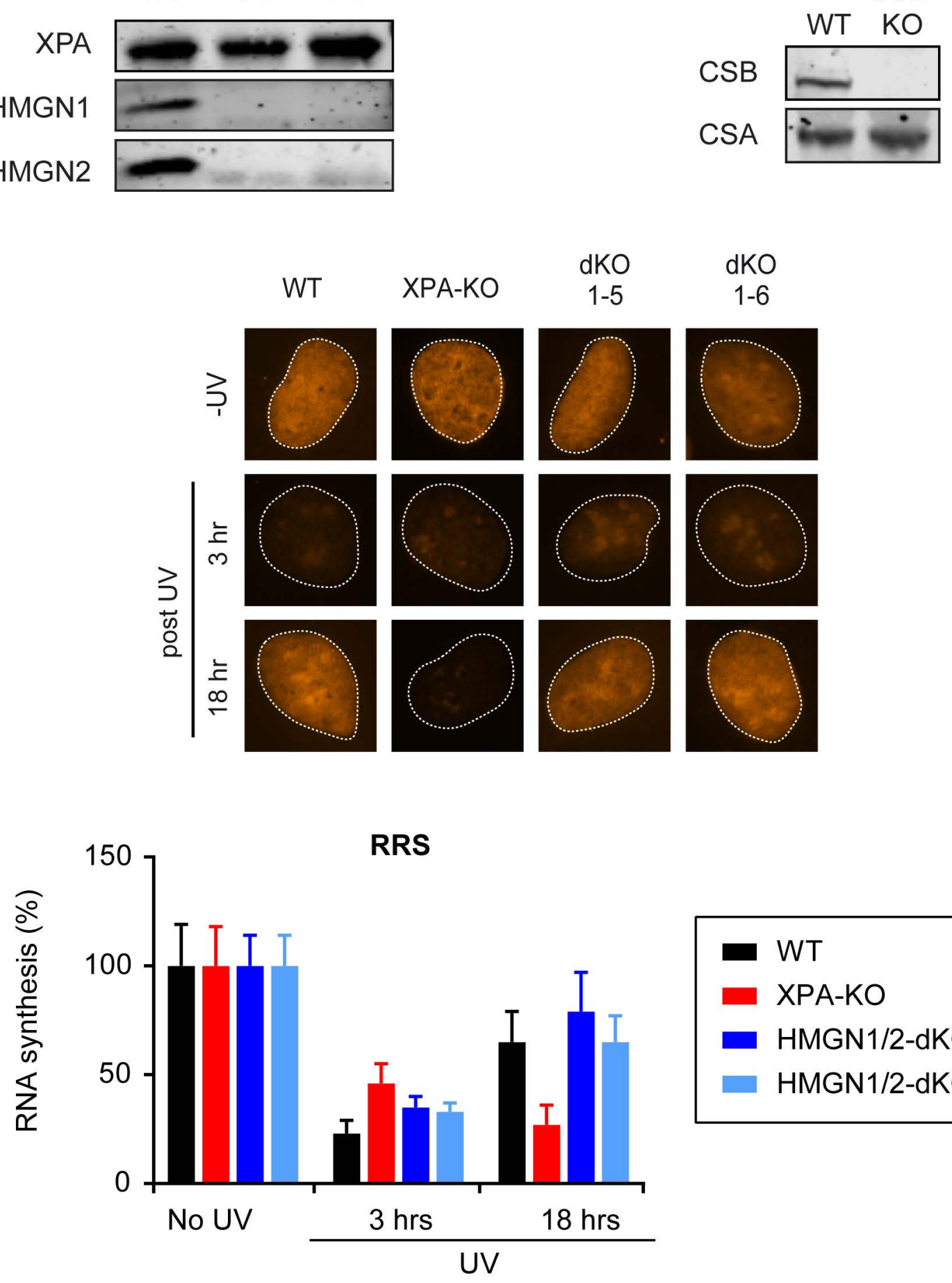

B

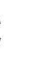

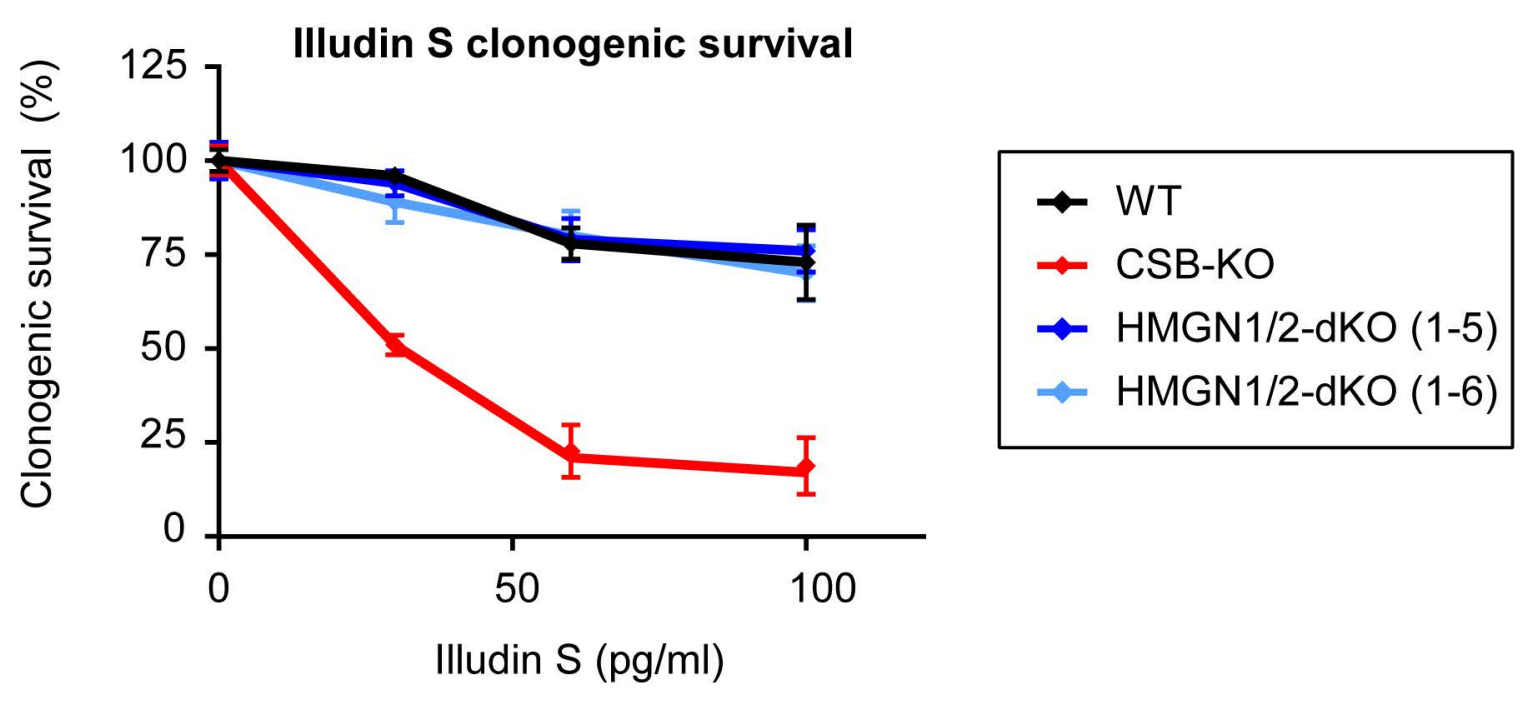


Figure 2. HMGN1 and HMGN2 double knockout does not impact human TCR. (a) Western blot analysis of U2OS WT and HMGN1/HMGN2-dKO clones or CSB-KO clone. (b) Representative microscopy images, and (c) Quantification of RRS after UV on the WT, XPA-KO, and HMGN1/HMGN2dKO cell lines. Data represent mean \pm SEM of five independent experiments. (d) Clonogenic Illudin $S$ survival of WT, CSB-KO, and HMGN1/HMGN2-dKO cell lines. Data represent mean \pm SEM of five independent experiments. Uncropped Western blot data is shown in the Supplementary Information file. 
bioRxiv preprint doi: https://doi.org/10.1101/835868; this version posted November 9, 2019. The copyright holder for this preprint (which Figure'as not certified by peer review) is the author/funder. All rights reserved. No reuse allowed without permission.

A

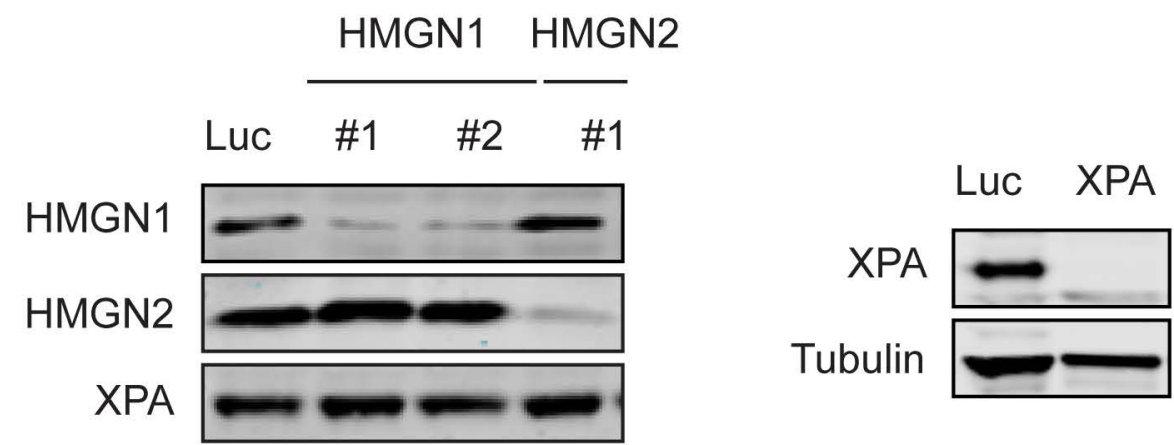

B

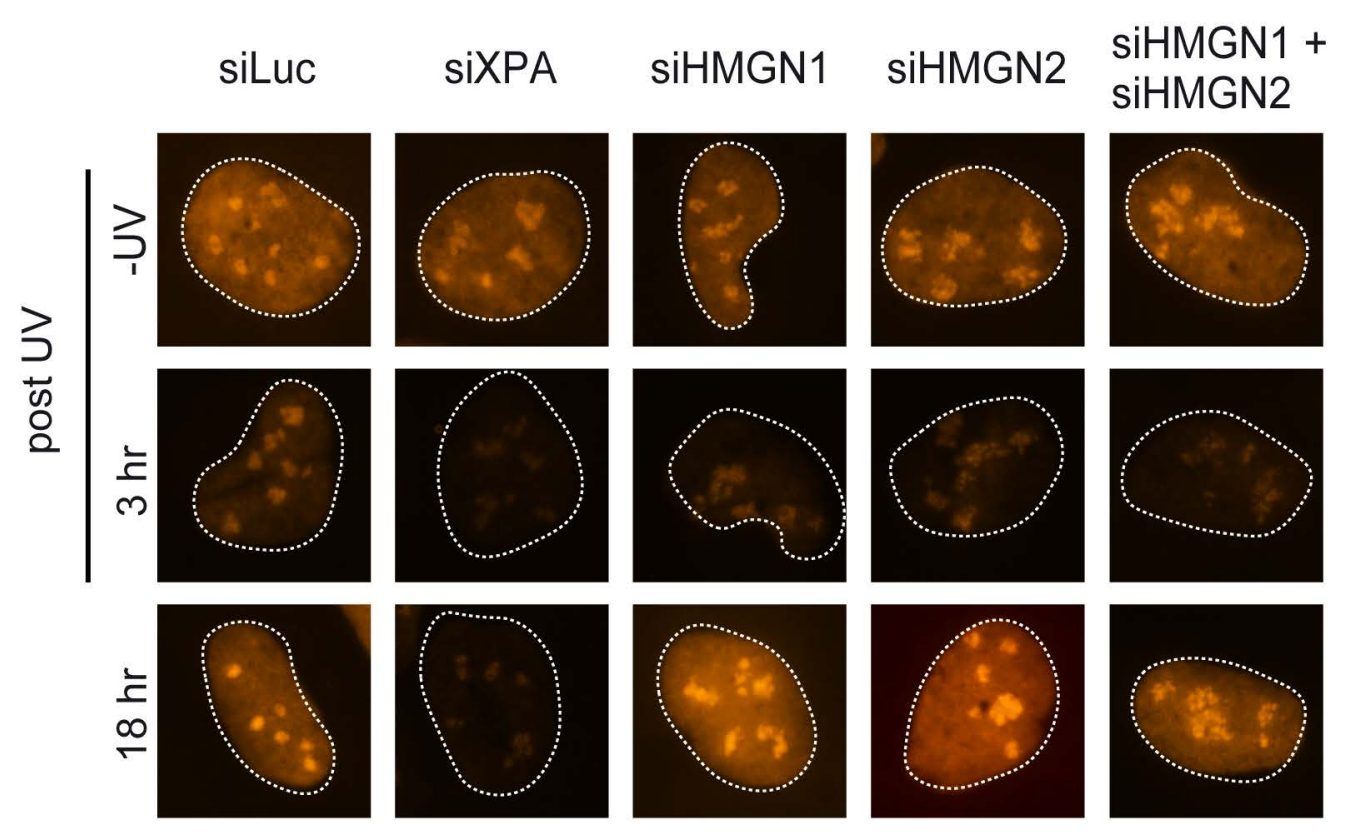

C
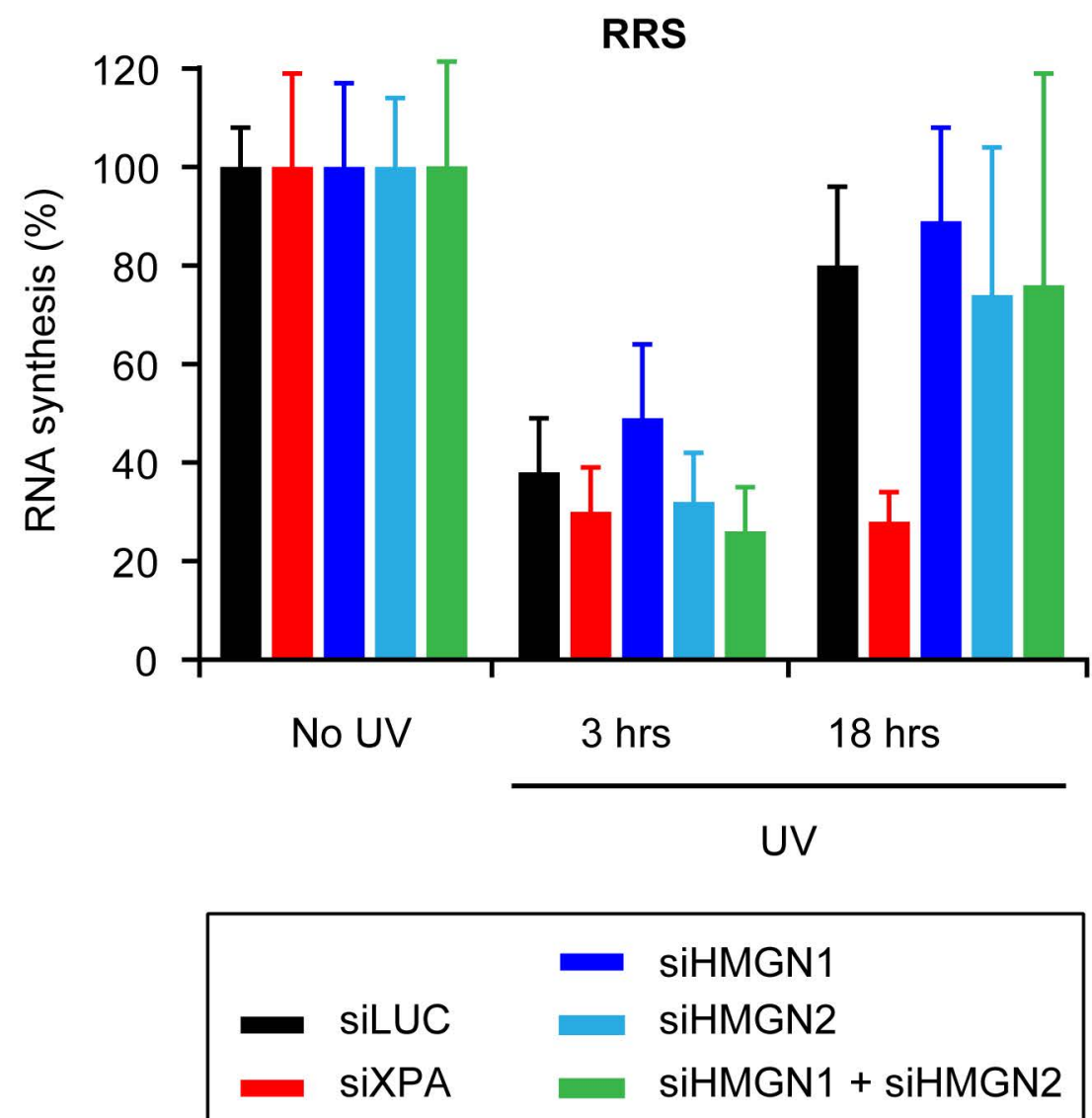
Figure 3. HMGN1 and HMGN2 knockdown does not impact human TCR. (a) Western blot analysis of U2OS cells transfected with the indicated siRNAs. (b) Representative microscopy images, and (c) Quantification of RRS after UV on U2OS cells transfected with the indicated siRNAs. Data represent mean \pm SEM of three independent experiments. Uncropped Western blot data is shown in the Supplementary Information file. 
Fighifre pyprint doi: https://doi.org/10.1101/835868; this version posted November 9, 2019. The copyright holder for this preprint (which

A
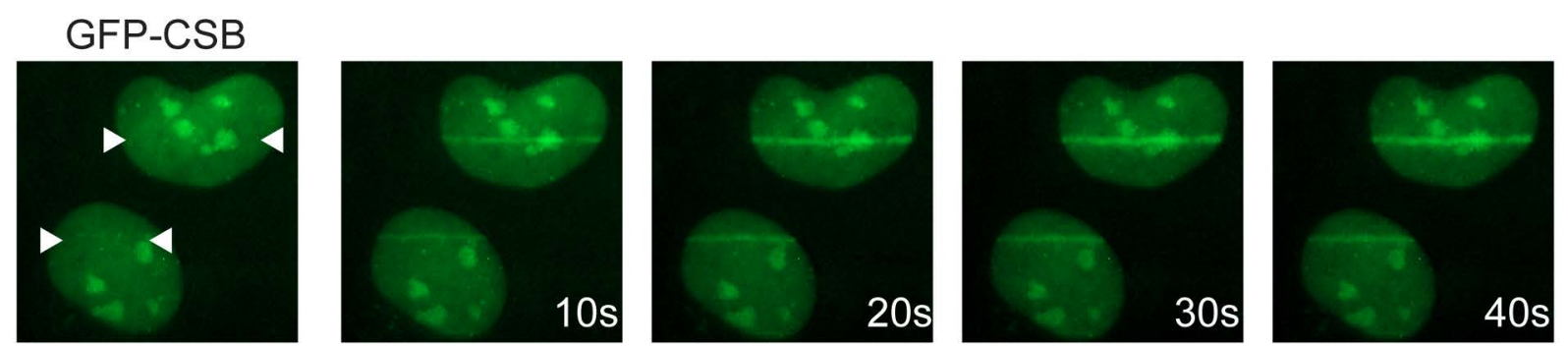

HMGN1-GFP
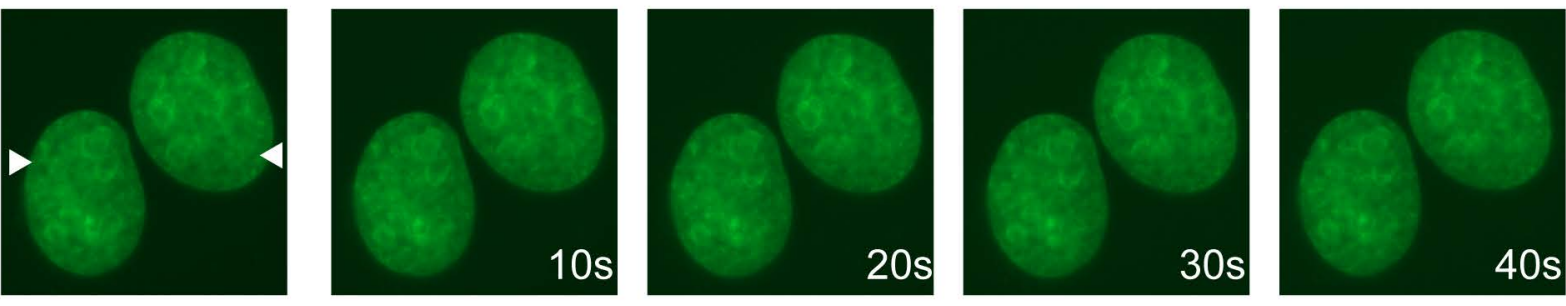

No UV

UV-C laser

B

\begin{tabular}{|c|c|c|c|c|c|}
\hline $\begin{array}{r}\text { CSB-KO } \\
+ \text { GFP-CSB }\end{array}$ & Input & & & GFP- & \\
\hline UV-C & $30 m$ & $4 h$ & - & $30 \mathrm{~m}$ & $4 \mathrm{~h}$ \\
\hline GFP-CSB & & & & $\longrightarrow$ & \\
\hline RNAPIIo & & & & $=$ & \\
\hline MGN1 & & & & & \\
\hline
\end{tabular}

C

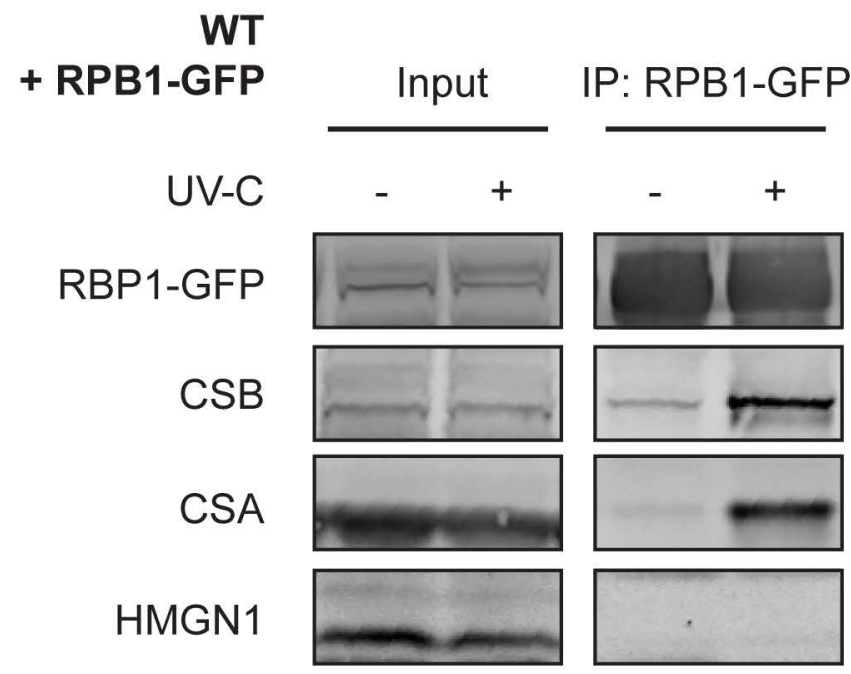


Figure 4. HMGN1 does not associate with the TCR complex. (a) Live-cell imaging on GFP-CSB or HMGN1-GFP after induction of UV-C-laser-induced DNA damage. The position of the laser track is indicated by white arrows. (b) Co-IP of GFP-CSB in unirradiated or UV-irradiated cells at the indicated time-points. (c) Co-IP of RPB1-GFP in unirradiated or UV-irradiated cells. Uncropped Western blot data is shown in the Supplementary Information file. 
Figure 5

bioRxiv preprint doi: https://doi.org/10.1101/835868; this version posted November 9, 2019. The copyright holder for this preprint (which

A

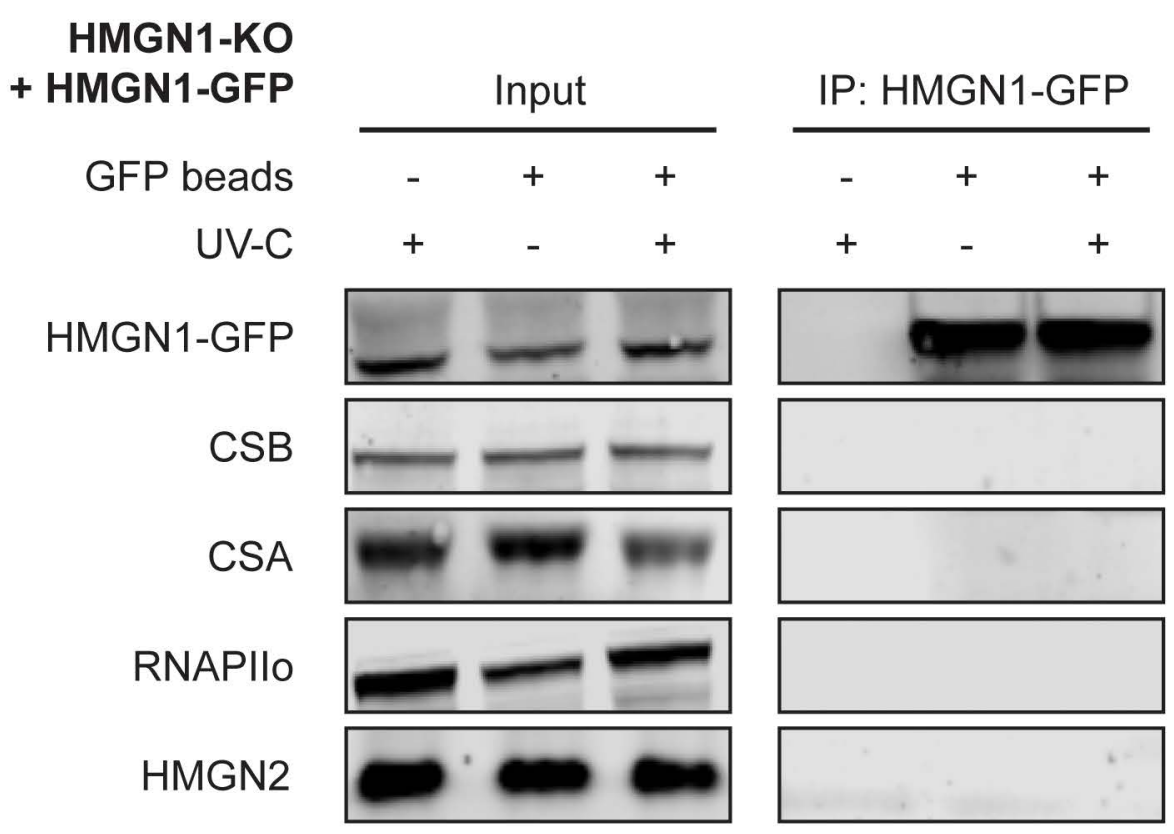

B

Input

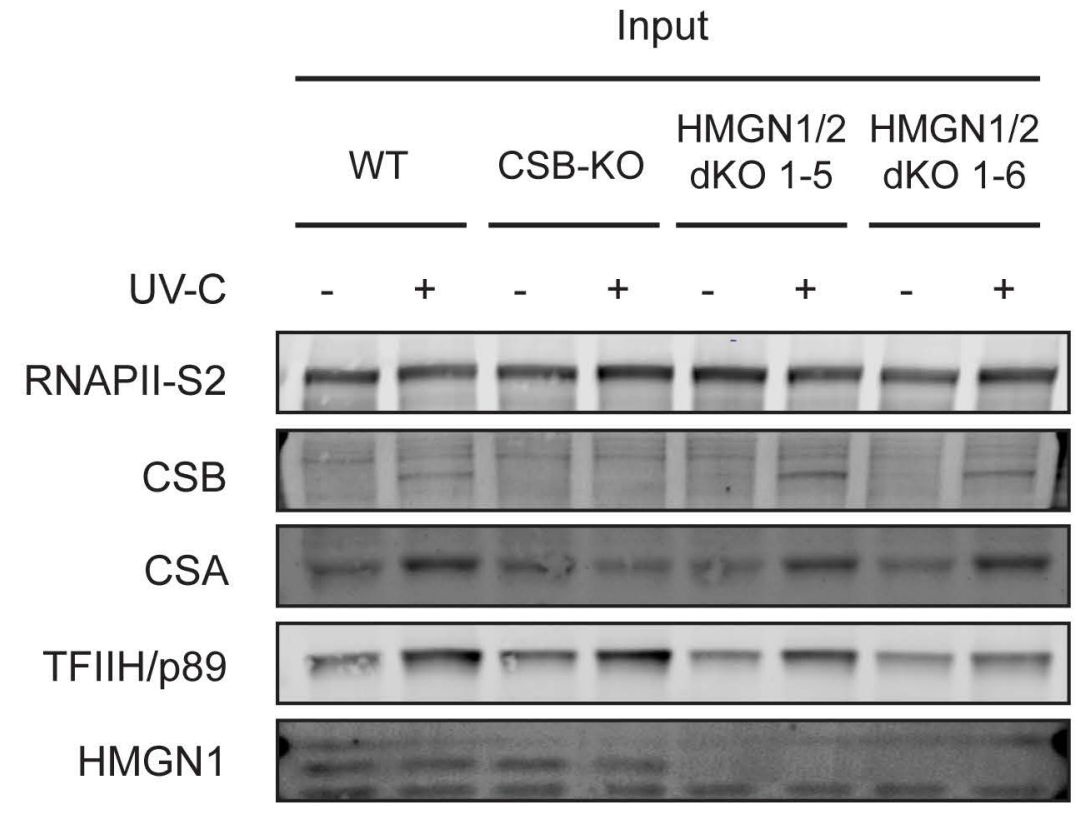

RNAPII-S2

CSB

CSA

TFIIH/p89

HMGN1
IP: RNAPIIO

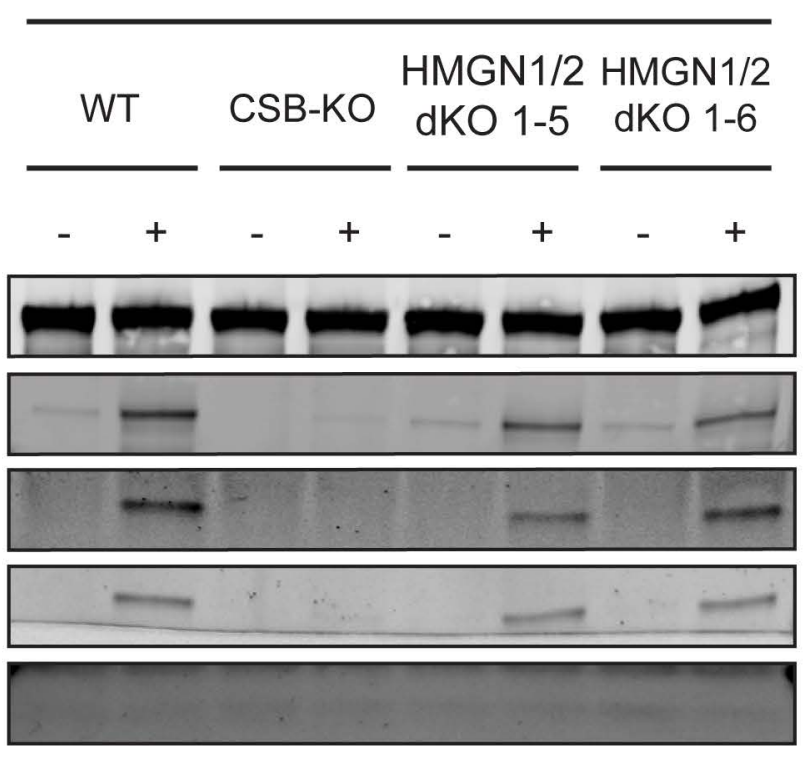


Figure 5. HMGN1 and HMGN2 are not required for TCR complex assembly. (a) Co-IP of HMGN1GFP in unirradiated or UV-irradiated cells at the indicated time-points. (b) Co-IP of endogenous RNAPIIo in WT, CSB-KO and HMGN1/HMGN2-dKO cell lines in unirradiated or UV-irradiated cells. Uncropped Western blot data is shown in the Supplementary Information file. 
Supplemental Figure 1. Alignment of HMGN proteins. (a) Sequence alignment of human and mouse HMGN1 proteins. (b) Sequence alignment of human HMGN1 and HMGN2 proteins. (C) Sequence alignment of human HMGN1, HMGN2, HMGN3, and HMGN4 proteins. All sequences were aligned with ClustalW. NLS = nuclear localization signal, NBD = nucleosome-binding domain, CHUD = chromatinunfolding domain. 